\title{
The peculiar supernova remnant CTB 80
}

\author{
F. Mavromatakis ${ }^{1}$, J. Ventura ${ }^{1,2,3}$, E. V. Paleologou ${ }^{2}$, and J. Papamastorakis ${ }^{1,2}$ \\ 1 University of Crete, Physics Department, PO Box 2208, 71003 Heraklion, Crete, Greece \\ 2 Foundation for Research and Technology-Hellas, PO Box 1527, 71110 Heraklion, Crete, Greece \\ 3 Max Planck Institut für Extraterrestrische Physik, 85741 Garching, Germany
}

Received 20 September 2000 / Accepted 2 March 2001

\begin{abstract}
Deep CCD exposures of the peculiar supernova remnant CTB 80 in the light of the $\mathrm{H} \alpha+[\mathrm{N}$ II], [S II] , $[\mathrm{O}$ II], and [O III] filters have been obtained. These images reveal significant shock heated emission in the area of the remnant. An extended bright diffuse nebula in the south-east part of CTB 80 overlaps soft X-ray emission from ROSAT but it does not appear to be related to the remnant under study. New diffuse and filamentary structures are detected to the south, south-east, and north of PSR 1951+32, most likely associated with CTB 80. Especially, the sulfur line image shows emission in the north along the outer boundary of the IRAS and HI shells. The [O III] emission is filamentary, suggesting shock velocities greater than $100 \mathrm{~km} \mathrm{~s}^{-1}$ but its spatial extent is quite limited. Lower shock velocities are expected in the north and north-east areas of the remnant, since [O II] emission is present, while [O III] line emission is not detected. The comparison between the [O III] and [O II] line images further suggest the presence of significant inhomogeneities in the interstellar medium. The flux-calibrated images do not indicate the presence of incomplete recombination zones, and we estimate that the densities of the preshock clouds should not exceed a few atoms per $\mathrm{cm}^{3}$. The typical projected angular widths of the observed filaments are $\sim 30^{\prime \prime}$. Typical surface brightness values of the long [O III] filament in the south are $\sim 1210^{-17} \mathrm{erg} \mathrm{s}^{-1} \mathrm{~cm}^{-2} \operatorname{arcsec}^{-2}$ while the [O II] image is characterized by fluxes of $10-2010^{-17} \mathrm{erg} \mathrm{s}^{-1} \mathrm{~cm}^{-2} \operatorname{arcsec}^{-2}$. The area covered by the optical radiation along with the radio emission at $1410 \mathrm{MHz}$ suggest that CTB 80 occupies a larger angular extent than was previously known.
\end{abstract}

Key words. ISM: general - ISM: supernova remnants - ISM: individual objects: CTB 80

\section{Introduction}

Downes (1970), almost 30 years ago, proposed that the radio source CTB $80(\mathrm{G} 69.0+2.7)$ was a supernova remnant. However, confirmation came only in 1974 when observations of total and polarized intensity of a number of radio sources were performed by Velusamy \& Kundu (1974). The non-thermal emission and the strong polarization of the source thus offered further support to the initial claim, and established the source's peculiar radio morphology. The radio images show the presence of a $\left(\sim 1^{\prime}\right)$ compact central source (spectral index $\sim 0.0$ for $S_{\nu} \sim \nu^{-\alpha}$ ), a plateau to the east of the compact core extending for $\sim 10^{\prime} \times 6^{\prime}$ (spectral index $\sim 0.3$ ) and three large-scale structures extending, roughly, to the north, to the east and to the south-west (spectral index $\sim 0.8$, Angerhofer et al. 1981; Strom \& Stappers 2000). The source, depending upon the frequency of observation, displays a different morphology, while the polarization is quite strong over a large part of the radio emission. Radio observations in

Send offprint requests to: F. Mavromatakis, e-mail: fotis@physics.uoc.gr
1984 and 1985 led Strom (1987) to propose that the radio properties of the central compact core indicated the presence of a central neutron star. Interest in this source intensified following the discovery of a $39.5 \mathrm{~ms}\left(\sim 10^{5} \mathrm{yr}\right.$ old) radio pulsar at the center of the radio compact core (Clifton et al. 1987). Shortly after the pulsar discovery, Fesen et al. (1988) reported the detection of a shell of infrared emission correlated with the radio lobes of CTB 80 . The IRAS shell centered $30^{\prime}$ east of the pulsar, and characterized by a diameter of $\sim 1^{\circ}$, is open in the south-west and has a higher surface brightness in the $60 \mu \mathrm{m}$ wavelength regime than in the $100 \mu \mathrm{m}$ regime. These properties led the authors to suggest that the presumably fast, moving pulsar caught up with the decelerating supernova shell and, through the pressure of its relativistic wind broke off the southwestern portion of the HI shell, deforming the magnetic field structure, and producing the peculiar south-west protrusion in the radio emission. This suggestion is further supported by the detection of an expanding HI shell by Koo et al. (1990). The HI shell is clumpy (Koo et al. 1993), its south-west part is also open 
and is very well correlated with the infrared shell. The distance to CTB 80 is approximately $2 \mathrm{kpc}$, although distances in the range of $1.5-2.5 \mathrm{kpc}$ cannot be excluded (Koo et al. 1993; Strom \& Stappers 2000). Major characteristics of the remnant in X-ray wavelengths are the low count rate, and its small spatial extent (Wang \& Seward 1984; Safi-Harb et al. 1995). The ROSAT data show that the soft X-ray flux is confined to the area around the pulsar $\left(\sim 11^{\prime} \times 5^{\prime}\right)$ while the medium and hard X-ray flux show extended emission east, south-east of the pulsar. The spectrum indicates an average photon power-law index of $\sim 1.8$. The column density was not well determined due to the low X-ray counting statistics and was found to lie in the range of $10^{21}-10^{22} \mathrm{~cm}^{-2}$ (Safi-Harb et al. 1995). The authors also studied the temporal behavior of the central source and concluded that the pulsed fraction has a significant dependence on the energy.

Several optical observations have been performed, though the majority of them have focused on the area in the immediate neighbourhood of the pulsar (e.g. Blair et al. 1988; Whitehead et al. 1989; Hester \& Kulkarni 1989). Wide field red plates taken by van den Bergh (1980) in $\mathrm{H} \alpha$ and $[\mathrm{S} \mathrm{II}]$ showed faint, patchy nebulosity. Blair et al. (1984) performed interference filter observations in $\mathrm{H} \alpha+[\mathrm{N} \mathrm{II}]$ of two $30^{\prime} \times 30^{\prime}$ areas located south-west and north-east of the pulsar. The area around the pulsar was also observed in [O III] but no emission was detected apart from the nebulosity surrounding the pulsar. Spectra taken at two restricted areas in the south-west and the northeast indicate moderate absorption and shock heating.

In this work we present the first comprehensive CCD imaging in $\mathrm{H} \alpha+[\mathrm{N} \mathrm{II}]$, [S II], [O II] and [O III], of the area around CTB 80, covering a wide field of $\sim 2^{\circ} \times 2^{\circ}$, at a resolution of $4^{\prime \prime}-5^{\prime \prime}$. These new optical images reveal a rich network of filamentary and diffuse structures allowing for a better comparison with existing radio, infrared, and Xray data. Information about the observations and the data reduction is given in Sect. 2, while in Sect. 3 we describe the observed morphology in the various filters. Finally, in Sect. 4 we discuss the physical properties of the remnant, its positional relation, and interaction with the interstellar medium (ISM).

\section{Observations}

\subsection{Optical images}

The optical images of CTB 80 were obtained with the $0.3 \mathrm{~m}$ telescope at Skinakas Observatory. The remnant was observed in July 9, July 10, and October 13, 1999. Two different CCDs were used during these observations. The first, was a $1024 \times 1024$ Thomson CCD which resulted in a $69^{\prime} \times 69^{\prime}$ field of view and an image scale of $4^{\prime \prime} .12$ per pixel. The second was a $1024 \times 1024$ Site CCD which had a larger pixel size resulting in a $89^{\prime} \times 89^{\prime}$ field of view and an image scale of $5^{\prime \prime} .21$ per pixel. Our aim was to cover the whole field defined by the faintest radio contours at $1410 \mathrm{MHz}$ (Mantovani et al. 1985). Given the large extent of the remnant, we performed several
Table 1. Interference filter characteristics

\begin{tabular}{lll}
\hline Filter & $\begin{array}{l}\text { Wavelength }^{\mathrm{a}} \\
(F W H M)(\AA)\end{array}$ & $\begin{array}{l}\text { Line }(\%) \\
\text { contributions }\end{array}$ \\
\hline $\mathrm{H} \alpha+[\mathrm{N} \mathrm{II}]$ & $6555(75)$ & $100,100,100^{\mathrm{b}}$ \\
\hline$[\mathrm{S} \mathrm{II}]$ & $6708(27)$ & $100,18^{\mathrm{c}}$ \\
\hline$[\mathrm{O} \mathrm{II}]$ & $3727(28)$ & $100,100^{\mathrm{d}}$ \\
\hline$[\mathrm{O} \mathrm{III}]$ & $5005(28)$ & 100 \\
\hline Cont red & $6096(134)$ & - \\
\hline Cont blue & $5470(230)$ & - \\
\hline
\end{tabular}

a Wavelength at peak transmission for $f / 3.2$.

b Contributions from $\lambda 6548,6563,6584 \AA$.

c Contributions from $\lambda 6716,6731 \AA$.

d Contributions from $\lambda 3727,3729 \AA$.

pointings in order to have the best possible coverage of the field. At each pointing the relevant field was observed for $1800 \mathrm{~s}$ at two different instances, i.e. the total exposure time was $3600 \mathrm{~s}$. Consequently, overlapping areas have an effective exposure time of 7200 s. Every field was projected to a common origin before any image combination. The astrometric solutions were calculated with the aid of the Hubble Space Telescope Guide Star catalogue. The characteristics of the interference filters used during the observations are listed in Table 1. All coordinates in this work refer to epoch 2000.

Standard IRAF and MIDAS routines were used for the reduction of the data. Individual frames were bias subtracted and flat-field corrected using well-exposed twilight flat-fields. The spectrophotometric standard stars HR 7596, HR 7950, HR 8634, and HR 718 were used for flux calibration.

\section{Results}

\subsection{The $H \alpha+[N I I]$ and [SII] line emission}

The field in the area of CTB 80 contains both filamentary and diffuse structures. The nebulosity around the pulsar at $\alpha \simeq 19^{\mathrm{h}} 53^{\mathrm{s}}$ and $\delta \simeq 32^{\circ} 53^{\prime}$ is clearly seen in Fig. 1 as a slightly extended "star-like" object situated in a $10^{\prime} \times 10^{\prime}$ patch of diffuse $\mathrm{H} \alpha+[\mathrm{N}$ II] emission. North of PSR $1951+32$, several small scale structures are detected in the $[\mathrm{SII}]$ image (Fig. 2). They are nicely correlated with the north radio ridge (Fig. 3) as well as with the north part of the $I(60 \mu \mathrm{m}) / I(100 \mu \mathrm{m})$ map of Fesen et al. (1988, hereafter FSS) while their relation to the $\mathrm{H} \alpha$ emission ([S II $] / \mathrm{H} \alpha \sim 1.0$ ) further suggests that we observe shocked material associated with the remnant CTB 80. To the west of the pulsar and towards the south, we find the known network of filaments (position I in Fig. 1, see also Fig. 1a of Blair et al. 1984). In this area, diffuse emission is seen to the west of the bright filament at $\alpha \simeq 19^{\mathrm{h}} 51^{\mathrm{m}}$ and $\delta \simeq 32^{\circ} 32^{\prime}$ (position II), lying just outside the faintest radio contours at $1410 \mathrm{MHz}$ (Mantovani et al. 1985). The typical surface brightness fluxes measured in the $\mathrm{H} \alpha+[\mathrm{NII}]$ and $[\mathrm{SII}]$ images lie in 


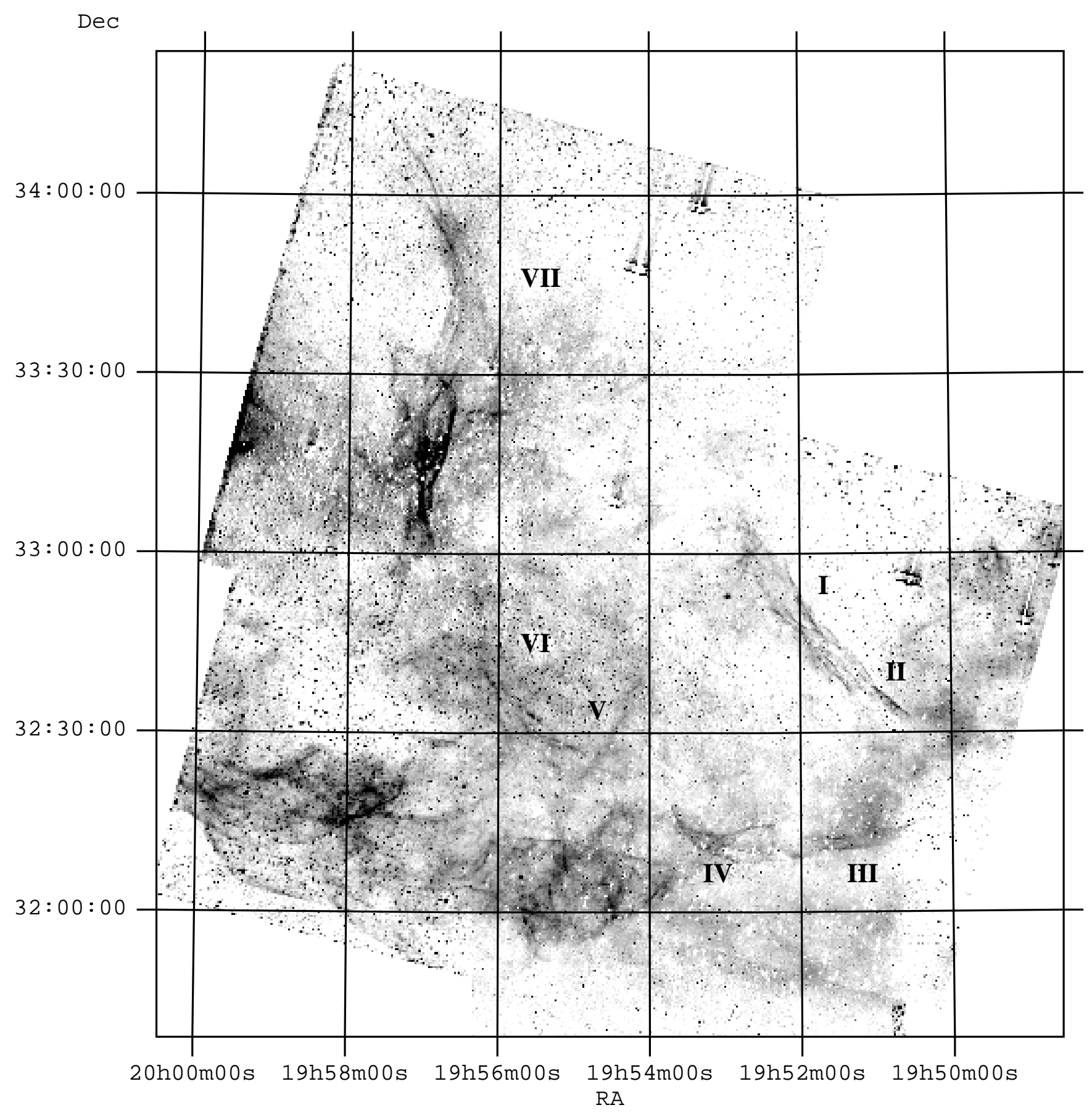

Fig. 1. CTB 80 imaged in the $\mathrm{H} \alpha+[\mathrm{N}$ II $]$ filter. The image has been smoothed to suppress the residuals from the imperfect continuum subtraction. Here and in all subsequent figures north is up, east to the left and the coordinates refer to epoch 2000 . Shadings run linearly from 0.0 to $7010^{-17} \mathrm{erg} \mathrm{s}^{-1} \mathrm{~cm}^{-2} \operatorname{arcsec}^{-2}$. The line segments seen near overexposed stars in this figure and the next figures are due to the blooming effect

the range of $30-4010^{-17} \mathrm{erg} \mathrm{s}^{-1} \mathrm{~cm}^{-2} \operatorname{arcsec}^{-2}$ and 5$1210^{-17} \mathrm{erg} \mathrm{s}^{-1} \mathrm{~cm}^{-2} \operatorname{arcsec}^{-2}$, respectively. South of the radio contours and at positions III and IV two patches of emission are detected running along the west-east direction at $\delta \simeq 32^{\circ} 10^{\prime}$. Faint emission, at the same declination, appears to fill the gap between these two locations and might indicate their physical relation. The optical radiation at location IV displays filamentary structures of projected widths $\sim 10^{\prime \prime}$ to $30^{\prime \prime}$. The structure seems to curve towards the north, north-east. The overall appearance of these structures in the [S II] image is less filamentary.

The bright diffuse emission to the south-east of position IV occupies an area of $\sim 30^{\prime} \times 18^{\prime}$ and its relation to CTB 80 is not certain. A search in the SIMBAD database around $\alpha \simeq 19^{\mathrm{h}} 55^{\mathrm{m}}$ and $\delta \simeq 32^{\circ} 05^{\prime}$ did not reveal any previously known extended object, although diffuse emission is seen in the POSS plates. North of this 


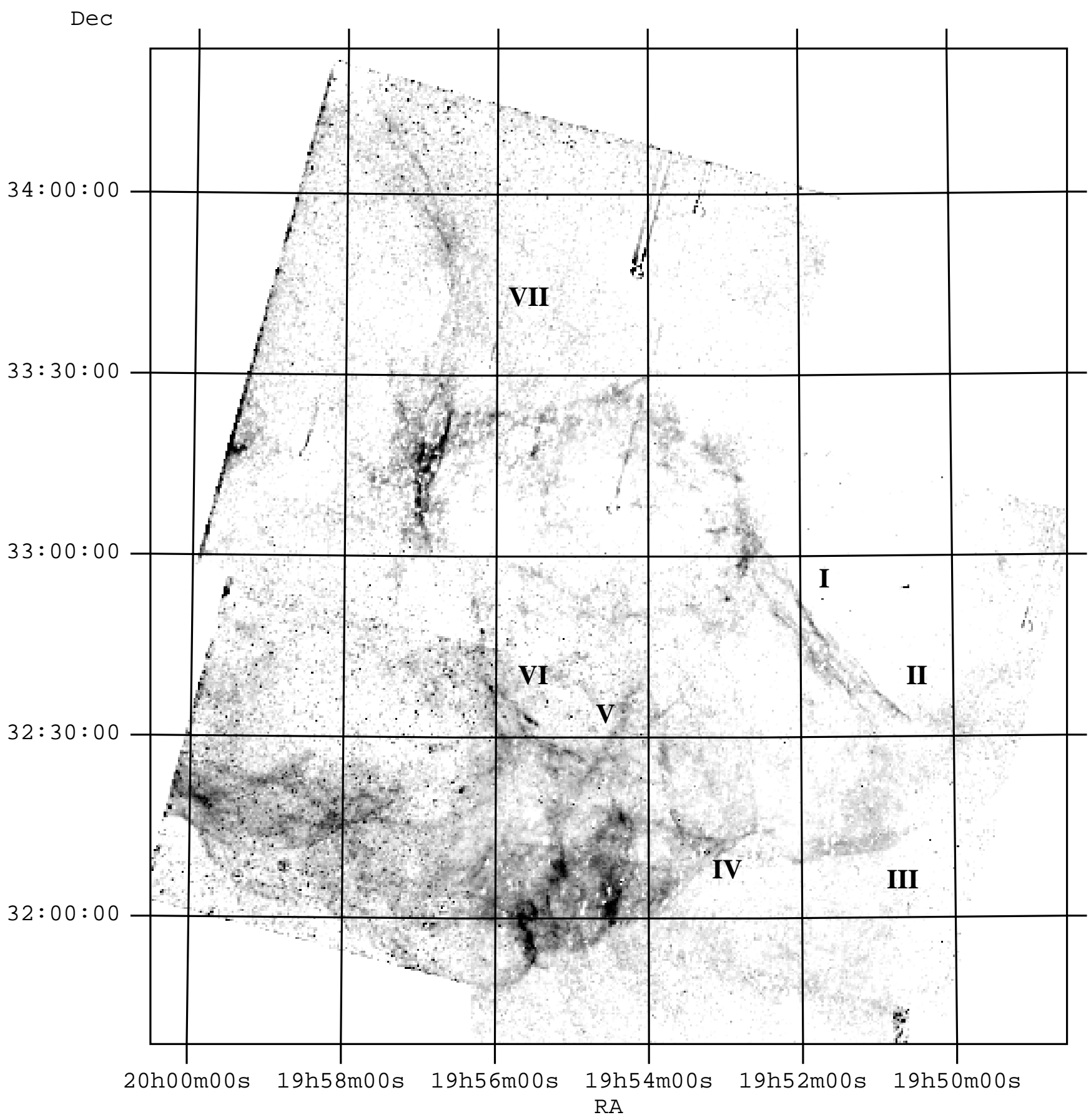

Fig. 2. CTB 80 imaged in the $[\mathrm{S}$ II] filter. The image has been smoothed to suppress the residuals from the imperfect continuum subtraction. Shadings run linearly from 0.0 to $2010^{-17} \mathrm{erg} \mathrm{s}^{-1} \mathrm{~cm}^{-2} \operatorname{arcsec}^{-2}$

extended structure, we come across two elongated structures at positions V and VI (Figs. 1, 2). The first one is oriented in the SE to NW direction and is characterized by a length of $\sim 20^{\prime}$ and typical thickness of $\sim 30^{\prime \prime}$ while the second is oriented along the SW to NE direction and extends for $\sim 33^{\prime}$. The thickness of this structure is $\sim 1^{\prime}$ and its position coincides with the outer south part of the $I(60 \mu \mathrm{m}) / I(100 \mu \mathrm{m})$ map of FSS. The positions of these locations ( $\mathrm{V}$ and $\mathrm{VI}$ ) completely overlap the radio contours (eastern ridge) at $1410 \mathrm{MHz}$ (Mantovani et al. 1985). At the NE boundary of the radio contours the known Lynd's Bright Nebula 156 (Lynds 1965) is found, which is quite bright in both the $\mathrm{H} \alpha+[\mathrm{N} \mathrm{II}]$ and $[\mathrm{S} \mathrm{II}]$ filters. Part of LBN 158 is seen to the west of LBN 156 at the west edge of our field. Interestingly, at the north end of LBN 156 we see two thin arcs convex to the west extending further to the north for $\sim 38^{\prime}$ (location VII). These two arcs have a projected thickness of $\sim 30^{\prime \prime}$ and cross each 


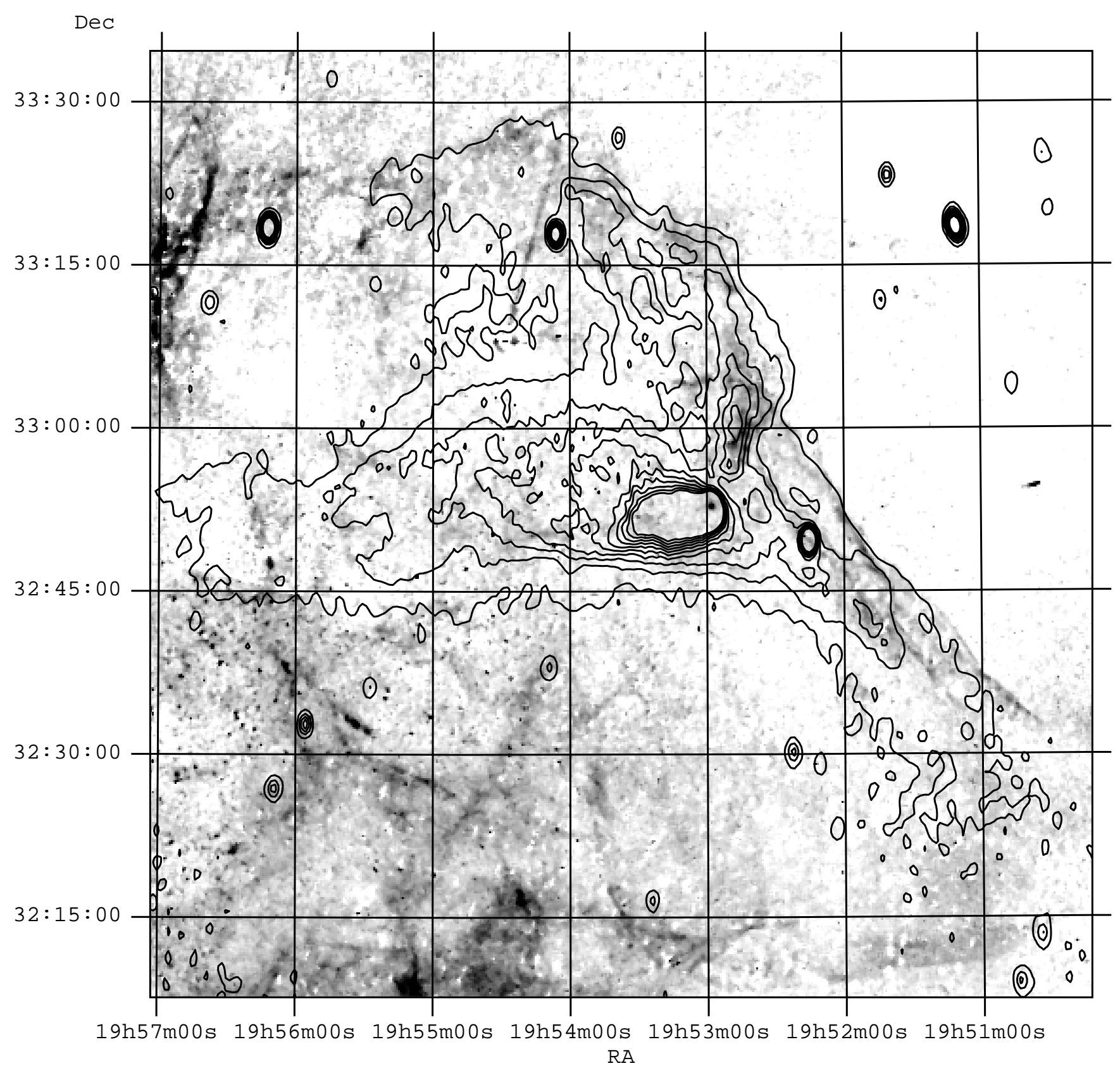

Fig. 3. The $49 \mathrm{~cm}$ radio contours (Strom \& Stappers 2000) are overlaid to the [S II] image shown in Fig. 2. The correlation of the small scale structures in the north is evident. The known filaments in the south-west are also seen. The radio contours are drawn linearly from 0.004 to $0.14 \mathrm{Jy} /$ beam

other at $\alpha \simeq 19^{\mathrm{h}} 56^{\mathrm{m}} 30^{\mathrm{s}}$ and $\delta \simeq 33^{\circ} 50^{\prime}$, while the crossing area is rather extended $\left(10^{\prime} \times 4^{\prime}\right)$. The arcs are also seen in the $[\mathrm{S} \mathrm{II}]$ image but are less prominent.

\subsection{The [O III] $\lambda 5007 \AA$ line emission}

The morphology of the area around CTB 80 in [O III] (see Fig. 4) is quite different from that seen in the $\mathrm{H} \alpha+[\mathrm{N} \mathrm{II}]$ and [S II] images. The field is relatively clean of diffuse, patchy emission while a few filamentary structures are seen. The first filament is detected SW of the pulsar and has a length of $\sim 3^{\prime}$ and a projected thickness of $\sim 25^{\prime \prime}$. It is located at position I where the corresponding $\mathrm{H} \alpha+[\mathrm{N}$ II] flux is lower by a factor of $\sim 2$ than the flux of the neighbouring filaments. Blair et al. (1984) did not detect any [O III] emission in their "SW" spectrum since it was taken $\sim 11^{\prime}$ away. Another [O III] filament which has an $\mathrm{H} \alpha+[\mathrm{N}$ II] and [S II] counterpart is located at $\alpha$ $\simeq 19^{\mathrm{h}} 50^{\mathrm{m}} 30^{\mathrm{s}}$ and $\delta \simeq 32^{\circ} 34^{\prime}$ (our position II, position "SSW" of Blair et al. 1984). Around $\alpha \simeq 19^{\mathrm{h}} 50^{\mathrm{m}}$ and $\delta \simeq 32^{\circ} 15^{\prime}$ we find a filament which is $\sim 12^{\prime}$ long and $\sim 40^{\prime \prime}$ wide (position IIa in Fig. 4). A noteworthy feature 


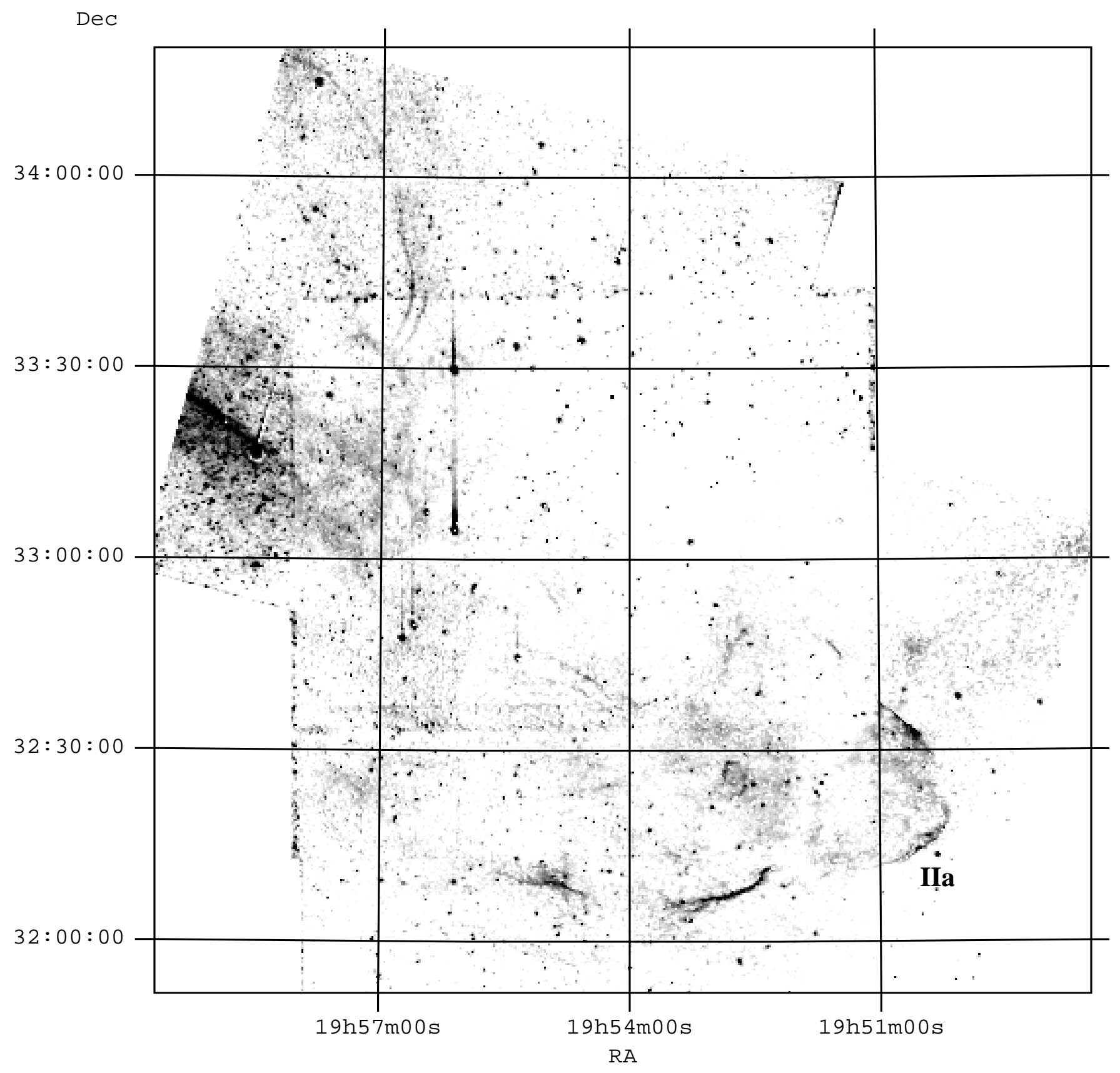

Fig. 4. CTB 80 imaged in the $[\mathrm{O}$ III $] \lambda 5007 \AA$ emission line. The image has been smoothed to suppress the residuals from the imperfect continuum subtraction. Shadings run linearly from 0.0 to $1010^{-17} \mathrm{erg} \mathrm{s}^{-1} \mathrm{~cm}^{-2} \operatorname{arcsec}^{-2}$

of this filament is its location along the outer radio contours, in the south, at $1410 \mathrm{MHz}$ (Mantovani et al. 1985). Emission from the low ionization lines of $\mathrm{H} \alpha+[\mathrm{N} \mathrm{II}]$, [S II] and [O II] (Figs. 1, 2, 5) is only partially correlated with this [O III] emission. To the south of position IV a $\sim 17^{\prime}$ long [O III $]$ filamentary structure is detected which has no obvious $\mathrm{H} \alpha+[\mathrm{N}$ II] counterpart. Its thickness ranges from $\sim 30^{\prime \prime}-60^{\prime \prime}$. This structure is $\sim 14^{\prime}$ away to the south of the outer $1410 \mathrm{MHz}$ radio contours. A peculiar [O III] structure, which has a shape similar to the number " 9 ", is found around $\alpha \simeq 19^{\mathrm{h}} 53^{\mathrm{m}}$ and $\delta \simeq 32^{\circ} 30^{\prime}$ just inside the outer radio contour at $1410 \mathrm{MHz}$. Finally, the last [O III] features present in the field are the arcs to the north of LBN 156 (position VII). However, only one of the arcs is seen for $\sim 38^{\prime}$ while the second one is seen for $\sim 10^{\prime}$.

\subsection{The low ionization line of [OII] $\lambda \lambda 3726,3729 \AA$}

The morphology of the field in [O II] (Fig. 5) is generally similar to that seen in $\mathrm{H} \alpha+[\mathrm{N}$ II] and [S II] but still, important diferences do exist. Some of the filaments seen in $\mathrm{H} \alpha+[\mathrm{N} \mathrm{II}], \mathrm{SW}$ of the pulsar (position I), have a counterpart in [O II] while others do not. Furthermore, in the position of an $\mathrm{H} \alpha$ filament, in the same area, we see in the 


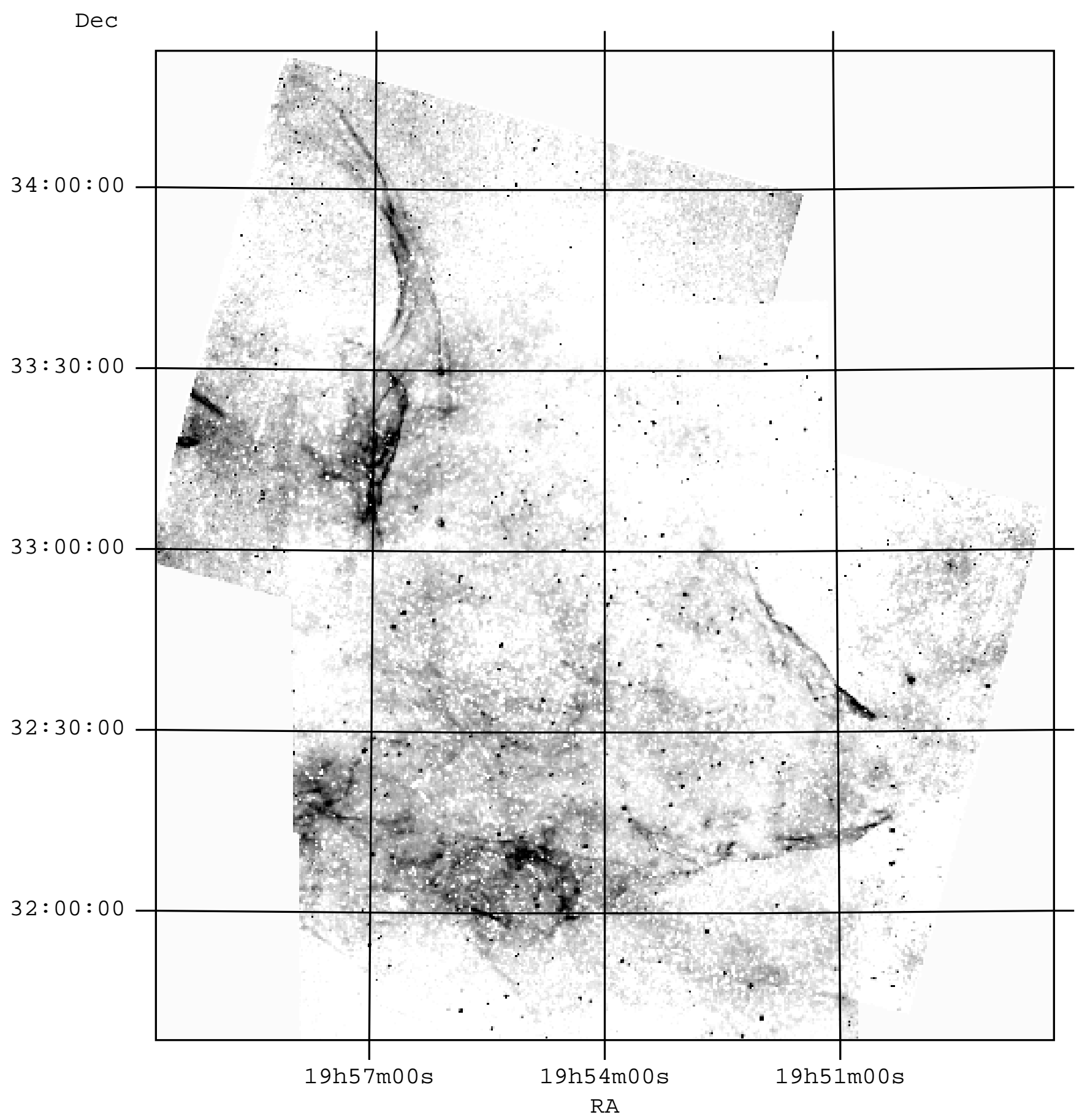

Fig. 5. CTB 80 imaged in the [O II] $\lambda 3726$ and $3729 \AA$ emission lines. The image has been smoothed to suppress the residuals from the imperfect continuum subtraction. Shadings run linearly from 0.0 to $2410^{-17} \mathrm{erg} \mathrm{s}^{-1} \mathrm{~cm}^{-2} \operatorname{arcsec}^{-2}$

[O II] image localized emission areas scattered along the general direction of the $\mathrm{H} \alpha+[\mathrm{N}$ II] filament. It is interesting to note that the filament seen in [O II] in the south of position I is better defined than in $\mathrm{H} \alpha+[\mathrm{N} \mathrm{II}]$. Additionally, we observe that the long [O III] filament (south of position IV) is seen as a series of localized emission "hills" in [O II]. The "valleys" (gaps) between the bright "hills" have typical lengths of the order of $0.2-0.4 \mathrm{pc}$. Also, while we detect $[\mathrm{O} \mathrm{II}]$ emission at position IV, there is practically no emission in [O III]. The extended diffuse structure in the south is also visible in [O II] while to its north, we observe less structured emission which is partially correlated with the $\mathrm{H} \alpha+[\mathrm{N}$ II] emission (positions V and VI). Finally, the bright nebula LBN 156 and the two arcs in the very north of our field are present and well correlated with the $\mathrm{H} \alpha+[\mathrm{N}$ II $]$ and $[\mathrm{S} \mathrm{II}]$ line emission. Deep exposures have been obtained from the area east of the two arcs and the results will be presented elsewhere (Mavromatakis et al. 2000). 
An interesting aspect concerning almost all of our line images is that the filaments in the west and the SW seem to define rather well the two sides of a triangle much like the shape of any closed, outer contour at $1410 \mathrm{MHz}$ (Mantovani et al. 1985).

\subsection{The ASCA spectral data}

We have analyzed public ASCA data of CTB 80 in order to have an accurate determination of the column density which would allow us to obtain estimates of the color excess, $E(B-V)$ and the average ISM density. An estimate of the color excess is desirable since current measurements refer to the pulsar neighbourhood (e.g. Hester \& Kulkarni 1989) while away from the pulsar the measurements are less certain (Blair et al. 1984). Determination of the hydrogen column density will provide us with an order of magnitude estimate of the ISM density around CTB 80 which can be compared with our estimates of the preshock cloud densities.

The source was observed in June 17, 1993 (sequence id 50037010) by the GIS and SIS detectors onboard the ASCA satellite (Halm 1996). Applying the strict selection criteria to the original data resulted in $25 \mathrm{ks}$ and 19 ks exposure time for the GIS and SIS detectors, respectively. The hardness ratios between the $0.8-2.0 \mathrm{keV}$, $2.0-6.0 \mathrm{keV}$ and the $6.0-12.0 \mathrm{keV}$ bands did not reveal any statistically significant spectral variations. Consequently, all photons within a $8^{\prime}$ radius from the center of the GIS intensity distribution were accumulated to create a source spectrum, while from the SIS data all photons within a rectangular area of $\sim 4^{\prime} \times 4^{\prime}$ were accumulated. An important point to be mentioned concerns the background subtraction. The available blank-sky background spectra are obtained from observations towards high galactic latitudes. However, CTB 80 is only $\sim 3^{\circ}$ away from the galactic plane, where a different spectral behavior of the sky background is expected. We have extracted a few observations from the ASCA database which were performed in the direction of CTB 80. The analysis showed that a thermal $(T \sim 0.59 \mathrm{keV})$ and a non-thermal component $(\alpha \sim 1.2)$ could fit the source-free spectra close to the galactic plane. Subsequently, these components were included in the fits of the SNR spectra by keeping the temperature and power-law index fixed at the aforementioned values.

We find that a simple power-model attenuated by the intervening column density provides a sufficient description of the data. The photon power-law index is $1.95_{-0.11}^{+0.15}$ and the column density is $2.9_{-0.5}^{+0.5} 10^{21} \mathrm{~cm}^{-2}$. The quoted errors refer to the $90 \%$ probability level for two parameters of interest $\left(\Delta \chi^{2}=4.61\right)$. In the case where we do not include the galactic plane background component, we find a photon power-law index of $1.88_{-0.05}^{+0.06}$ and a column density of $2.6_{-0.3}^{+0.3} 10^{21} \mathrm{~cm}^{-2}$. The two methods provide results that agree within the statistical errors due to the low counting statistics of CTB 80 . Nevertheless, the contribution of the galactic plane background should always be taken into account when analyzing source spectra near the galactic plane. We note here that our results are in good agreement with the results reported by Safi-Harb et al. (1995), however, the allowed range of the parameters is much narrower now.

Current measurements of the color excess suggest values in the range of $\sim 0.7-1$ (e.g. Blair et al. 1984, 1988). Assuming the relation $N_{\mathrm{H}}=6.8( \pm 1.6) 10^{21} \times E(B-$ $V) \mathrm{cm}^{-2} \mathrm{mag}^{-1}$ (Ryter et al. 1975), and the best determined $N_{\mathrm{H}}$ from the ASCA data, we derive a color excess of $0.38 \pm 0.10 \mathrm{mag}$. Clearly, the relation used is a statistical relation and deviations of 3-4 $\sigma$ could be justified. However, if the observed difference is true, then the X-ray measured color excess of $\sim 0.4$ will represent the amount of pure interstellar extinction. The additional $0.3-0.4$ mags of extinction would be due to local extinction around the core area. In any case, applying the interstellar reddening curve of Whitford (1958) as presented by Kaler (1976), an interstellar extinction $c$ of $0.57( \pm 0.15)$ is obtained. Finally, the average ISM density towards CTB 80 is $\sim 0.5 \mathrm{~cm}^{-3}$, assuming a distance of $2 \mathrm{kpc}$ (Koo et al. 1993; Strom \& Stappers 2000).

\section{Discussion}

\subsection{The $\mathrm{HI}$ and infrared shells}

FSS reported the detection of an infrared emitting shell in the area of CTB 80 . The shell posseses an angular diameter of $\sim 1^{\circ}$, is characterized by strong $60 \mu \mathrm{m}$ emission relative to the $100 \mu \mathrm{m}$ emission and is correlated with the radio emission. HI observations by Koo et al. (1990) revealed the presence of a clumpy shell, open in the south-west, expanding at $\sim 70 \mathrm{~km} \mathrm{~s}^{-1}$. They also estimated a density of the HI medium of $1.7 \mathrm{~cm}^{-3}$. Subsequent VLA HI observations by Koo et al. (1993) showed that the clumpy nature of the HI shell is caused by dense, fast moving HI clumps. These clumps preexisted the supernova explosion and were accelerated by the blast wave. The two shells (infrared and HI) are very well correlated, suggesting their association with CTB 80, while the smaller extent of the $\mathrm{HI}$ shell is probably due to the difficulty in distinguishing emission of the low radial velocity portion of the HI shell from galactic background emission (Koo et al. 1993).

The optical emission detected north, north-east of the pulsar position is rather well correlated with the IRAS ${ }^{1}$ ratio map of $I(60 \mu \mathrm{m}) / I(100 \mu \mathrm{m})$ of FSS as well as with the HI shell of Koo et al. (1990). The optical emission in this area lies inside the outer infrared (dashed) contour and outside the HI (dash-dotted) contour in Fig. 6. The solid contour corresponds to radio continuum emission at $49 \mathrm{~cm}$ (Strom \& Stappers 2000) while the three short lines in

1 The digital infrared data, originally presented by Fesen et al. (1988), were retrieved from

http://skyview.gsfc.nasa.gov 


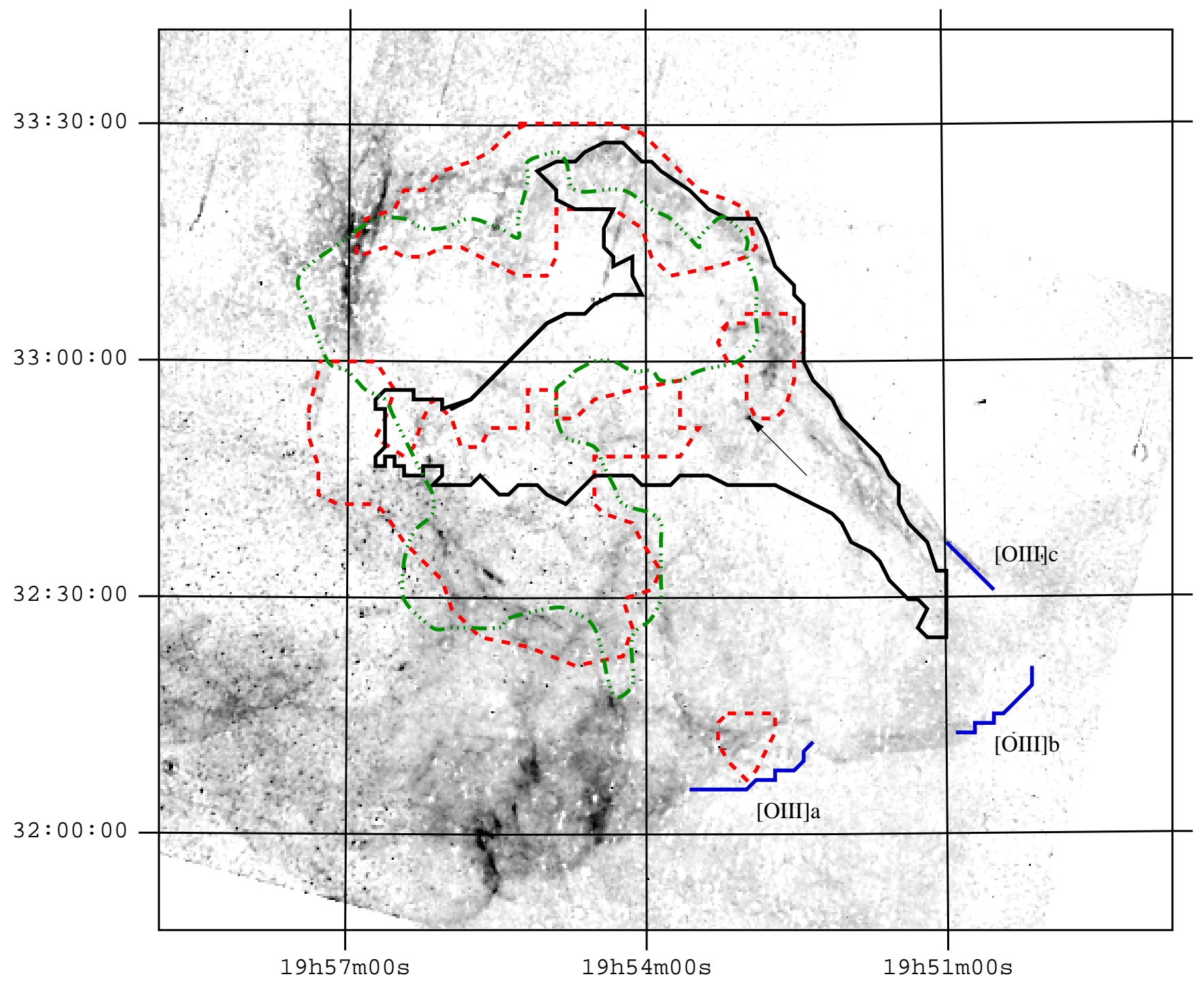

Fig. 6. CTB 80 imaged in the $[\mathrm{S}$ II] filter as a grey scale representation. The solid line outlines the $49 \mathrm{~cm}$ radio emission and the arrow points to the pulsar location. The dashed line delineates the infrared shell while the dash-dotted line delineates the HI shell. The short lines in the south labeled as [O III]a, b, c indicate the locations of [O III] filaments

the south, south-west indicate the positions of the prominent [O III] filaments. As seen from Fig. 6 there is also a good degree of correlation between HI, infrared and optical emission in the south, south-east (positions V and VI). The degree of correlation between the optical and infrared data increases when we consider the individual IRAS maps of e.g. the $60 \mu \mathrm{m}$ emission. Optical forbidden line emission at positions IV and V seem to be related to the $60 \mu \mathrm{m}$ emission. Based on this positional correspondence between the optical, HI and infrared emission, and the estimated flux ratio of $[\mathrm{S} \mathrm{II}] / \mathrm{H} \alpha \sim 0.7-1.0$ (extinction of $\sim 0.6$ asssumed) at these areas, it is proposed that the observed optical emission is indeed associated with CTB 80. The filamentary and diffuse structures in locations III, IV, V, and VI along with LBN 156 may be viewed as forming part of an almost continuous shell structure. This adds further support to FSS's previous suggestion that LBN 156 is also associated with CTB 80.

\subsection{The south-west area}

The calibrated flux images at positions I and II (Fig. 1) do not provide any strong evidence for incomplete recombination zones. The estimated values of $[\mathrm{O}$ III $] / \mathrm{H} \beta$ are found in the range of $1-3$ while a value of $\sim 7$ (the minimum extinction of 0.6 was assumed) is obtained at position IIa (Fig. 4). Raymond et al. (1988) report a maximum ratio of 6 for complete shock structures and shock velocities between 80 and $140 \mathrm{~km} \mathrm{~s}^{-1}$. Since we are dealing most likely with complete shocks, the results of Cox \& Raymond (1985) and Raymond et al. (1988) can be used to infer some basic shock properties. The presence of faint [O III] emission $\left(\sim 610^{-17} \mathrm{erg} \mathrm{s}^{-1} \mathrm{~cm}^{-2} \operatorname{arcsec}^{-2}\right)$ together with the high values of the $[\mathrm{O} \mathrm{II}] /[\mathrm{O} \mathrm{III}]$ ratio $(>3-8$, extinction of $\sim 0.6$ asssumed) suggest shock velocities between 85 and $120 \mathrm{~km} \mathrm{~s}^{-1}$, and most probably towards the high end of this range. Since the column density of a fully developed flow, roughly, scales as $10^{18.5} \times u_{\mathrm{s}, 100}^{2} \mathrm{~cm}^{-2}$ 
(Raymond et al. 1988), where $u_{\mathrm{s}, 100}$ denotes the shock velocity in units of $100 \mathrm{~km} \mathrm{~s}^{-1}$, we can set upper limits to the preshock cloud density. The column density is $N=\int_{0}^{l} n(l) \mathrm{d} l>n_{\mathrm{c}} l$, where $l$ is the projected thickness of a filament and $n_{\mathrm{c}}$ the preshock cloud density. A typical thickness of a filament at positions I and II is $910^{17} \mathrm{~cm}$ for a distance of $2 \mathrm{kpc}$ (Koo et al. 1993; Strom \& Stappers $2000)$, thus we obtain $n_{\mathrm{c}}<3.6 \times u_{\mathrm{s}, 100}^{2} \mathrm{~cm}^{-3}$. For shock velocities in the range of 85 and $120 \mathrm{~km} \mathrm{~s}^{-1}$, we get $n_{\mathrm{c}}<$ $2.6 \mathrm{~cm}^{-3}$ and $5.2 \mathrm{~cm}^{-3}$, respectively.

In neighbouring locations around positions I and II possible [O III] emission is below our detection limit while [O II] emission is detected well above the sky background. Since these two lines originate from the same element, it is rather likely that the shock velocity is less than $100 \mathrm{~km} \mathrm{~s}^{-1}$. In this case, the upper limit to the preshock cloud density becomes $3.6 \mathrm{~cm}^{-3}$. A possible explanation for the lower shock velocities could involve higher cloud densities by factors of 1.5-2.0 than the cloud densities where both [O II] and [O III] are detected. A different situation seems to hold for the [O III] filament at position IIa. It is partially correlated with [O II] emission while the degree of correlation is even smaller when the $\mathrm{H} \alpha+[\mathrm{N}$ II] and $[\mathrm{S} \mathrm{II}]$ images are examined. These morphological differences suggest the presence of inhomogeneities in the preshock medium. The low ionization lines are produced in areas of lower temperatures while the [O III] line emerges from higher temperature regions. The less hot areas are found at larger distances behind the shock front and consequently, at higher column densities. Inhomogeneities in the preshock clouds would mainly affect regions of higher column densities. At the same time, preshock cloud density variations would also affect the recombination zone through their effect on the cooling and recombination time scales (see Hester 1987 for more details).

\subsection{The bright [OIII] filament in the south}

In the south and close to position IV, a long [O III] filament is detected. Typical surface brightness values are of the order of $1210^{-17} \mathrm{erg} \mathrm{s}^{-1} \mathrm{~cm}^{-2} \operatorname{arcsec}^{-2}$. The same spatial location in the $[\mathrm{O} \mathrm{II}]$ image looks completely different where small size patches $\left(\sim 50^{\prime \prime} \times 30^{\prime \prime}\right)$ of emitting material are seen, instead of a well defined filament. According to Cox \& Raymond (1985) shock velocities greater than $100 \mathrm{~km} \mathrm{~s}^{-1}$ would be required to give rise to the observed $[\mathrm{O} \mathrm{II}]$ and $[\mathrm{O} \mathrm{III}]$ fluxes. Inhomogeneities and preshock density variations are probably the cause of the observed differences in morphology. The CCD images suggest angular length scales of $\sim 25^{\prime \prime}$ of these irregularities. An important issue related to the presence of this filament is whether or not it is associated with CTB 80. Even though, a negative correlation can not be formally excluded, a positive correlation may be more favorable given the shape, the spatial location and emission characteristics of this [O III] filament. In addition, we note that this filament is located along the outer boundary of a region of relatively strong
$60 \mu \mathrm{m}$ emission seen in the IRAS maps (Wheelock et al. 1994). A positive indentification would imply a larger extent than currently assumed and, consequently, a larger shock radius. New, more sensitive radio observations at $1410 \mathrm{MHz}$ would help to clarify this issue.

\subsection{The east area of CTB 80}

The east, south-east area of CTB 80 shows mainly diffuse optical radiation, even though a few filamentary structures are present. These are characterized by lengths of $\sim 60^{\prime \prime}-$ $110^{\prime \prime}$ and projected widths of $\sim 20^{\prime \prime}$. Assuming complete shock structures, shock velocities less than $100 \mathrm{~km} \mathrm{~s}^{-1}$ and a distance of $2 \mathrm{kpc}$ (Koo et al. 1993; Strom \& Stappers 2000), we find that the preshock cloud densities $n_{\mathrm{c}}$ should satisfy $n_{\mathrm{c}}<5 \mathrm{~cm}^{-3}$. Such shock velocities may be expected, according to the calculations of Cox \& Raymond (1985), since associated [O III] emission is not detected, while in one location $\left(\alpha=19^{\mathrm{h}} 55^{\mathrm{m}} 35^{\mathrm{s}}\right.$ and $\left.\delta=32^{\circ} 33^{\prime} 03^{\prime \prime}\right)$ [O II] is also detected well above the sky background. SafiHarb et al. (1995) analyzed ROSAT PSPC and HRI data taken from the field of CTB 80. The PSPC data show the presence of four extended irregular structures in the south-east apart from the emission observed around the pulsar. The whole enhancement is described as the conelike feature by Safi-Harb et al. (1995) and includes an elongated structure along the outer boundary of the PSPC in the south-east. The cone-like feature overlaps the optical emission at positions V and VI and the elongated structure partially overlaps the bright diffuse emission south of position IV. Given the strong sulfur line emission observed from this bright diffuse structure, it would be interesting to see if future radio observations would detect a thermal or non-thermal spectrum. However, at the moment, no firm conclusions can be drawn about the correlation between the optical and X-ray data.

\subsection{The nature and extent of CTB 80}

Although shell-like structures have been seen in HI, the infrared, and now in the optical, CTB 80 is not a common shell SNR. There is substantial and multifaceted evidence that PSR $1951+32$ has strongly interacted with and altered the remnant as is evident from its unusual radio appearance. It is necessary to proceed with care in attempting to reconstruct its evolution. As noted earlier (Sect. 4.2) the optical observations appear to support the suggestion of FSS and Koo et al. (1990) of a breakout of the SN shell south west of the pulsar, presumably through the action of a strong particle and electromagnetic wind, which has deformed the shell's magnetic field, and has given rise to the south western radio emission ridge.

The radio images at $1410 \mathrm{MHz}$ and $1720 \mathrm{MHz}$ show that faint emission extends for more than 1.5 in the northsouth and west-east directions. The optical data span from 32.2 to 33.5 in declination, while the span in right ascension is somewhat smaller. The new optical emission 
features detected in the south, south-east (positions IV, $\mathrm{V}$ and VI) seem to define an extended emission arc of large curvature, while the optical features detected in the north delinetate an emission arc of smaller curvature. This apparent discrepancy could be reconciled if we adopt the evolutionary scenario of CTB 80 proposed by Koo et al. (1993). According to this scenario, soon after the supernova explosion $(\sim 2000 \mathrm{yr})$ the blast wave broke out into a cavity in the south-east, while in the north, north-west the shock front propagated into a denser but clumpy interstellar medium. The pulsar was born with a westward velocity, and caught up the slower expanding shell after $\sim 10^{5}$ yr. Consequently, the optical structures at positions III, IV and VI may roughly define the south, south-east boundaries of a cavity seen in the HI data, while the structure at position $\mathrm{V}$ may be projected into the cavity area, or else be related to the cavity's foreground edge.

If this interpretation is correct, then the optical data suggest that the cavity must extend from declination $\simeq 33^{\circ} 10^{\prime}$ to $\simeq 32^{\circ} 10^{\prime}$. The correlation between the enhanced IRAS emission along the east cavity wall, and the optical radiation may suggest that the shock front encountered a dense medium. Note here that diffuse X-ray emission is mainly detected in this area (Safi-Harb et al. 1995) which may tie in with the presence of the HI cavity permeated by hot, low density gas (Koo et al. 1993). The secondary shock driven into these dense clouds allowed the production of $\mathrm{H} \alpha,[\mathrm{N}$ II] , and [S II] radiation but its velocity was not high enough to allow for the production of [O III] radiation, i.e. the shock velocity is probably less than $\sim 70 \mathrm{~km} \mathrm{~s}^{-1}$ (Cox \& Raymond 1985; Hartigan et al. 1987). However, [O III] filamentary emission (positions I, II, IIa, and III) is clearly detected in the south, south-west (Figs. 4 and 6). Consequently, shock velocities greater than $\sim 80 \mathrm{~km} \mathrm{~s}^{-1}$ are expected in these locations according to the calculations of Cox \& Raymond (1985). These areas are found to the south of the dense HI cloud detected by Koo et al. (1993) where the IRAS and HI emission is weak (FSS, Koo et al. 1990). The presence of shock heated filaments at positions I and II suggests that the injection of pulsar-generated relativistic particles (FSS) has a strong impact on the "break-out" portion of the remnant's south western segment which may have been compressed westward, possibly against preexisting "clouds". The rich network of filaments seen in the low ionization lines and the spatially limited but filamentary [O III] emission (positions I and II) on the leading edge of the south-west radio ridge indicate different shock velocities as well as inhomegenities in the interstellar medium (see also Koo et al. 1993).

Summarizing, we can propose the following. The optically emitting gas detected in the north in the [S II] filter delineates a circular structure which is very well correlated with the radio, infrared and HI radiation. The north part of the emission corresponds to the undisturbed part of the shock while in the south-east the accelerated shock front has reached the oposite walls of the HI cavity. The shock velocity into the cavity walls was sufficiently low not to allow for the production of [O III] emission while faster shocks have propagated towards the south, south-west. The pulsar relativistic wind interacts with the preexisting clouds, giving rise to a complex network of filaments along the south-west radio ridge.

\section{Conclusions}

$\mathrm{A} \sim 2^{\circ} \times 2^{\circ}$ area around the pulsar PSR $1951+32$ was observed in $\mathrm{H} \alpha+[\mathrm{N}$ II] , [S II], [O III] and [O II]. The low ionization line images of [S II] and [O II] show significant emission not before detected at locations in the south and southeast. The [S II] image also shows emission north of the pulsar, clearly, tracing the infrared shell and the north radio ridge. The morphology of the $\mathrm{H} \alpha+[\mathrm{N}$ II] and [S II] images is similar. The [O III] image appears rather faint in relation to the images in the lower ionization lines, probably reflecting the remnant's advanced age and low expansion velocity. The differences between the oxygen line images and the morphologies seen in the lower ionization images suggest the existence of density variations and inhomogenities in the preshock interstellar medium. Rough upper limits to the preshock density of the interstellar clouds are of the order of a few atoms per $\mathrm{cm}^{3}$ while the ASCA X-ray data suggest an average insterstellar medium density of $\sim 0.5 \mathrm{~cm}^{-3}$. Based on our upper limits, it is estimated that the density contrast between the ISM clouds and the ISM medium is less than $\sim 5$.

The absence of radio emission from the main body of the infrared and HI expanding shells is conspicuous and surprising, especially as these shells are reported to be very massive (Koo et al. 1990, 1993). The area covered by the $1410 \mathrm{MHz}$ radio emission and the optical radiation detected in the south and south-east indicate that the accelerated shock front has traversed the HI cavity and is interacting with its walls. More sensitive radio observations at $1410 \mathrm{MHz}$ would be required to establish the relation of the optical structures in the south of CTB 80, and the actual size of the remnant which is a crucial parameter for the further modeling of this remnant. An extended diffuse structure is detected in the south-east well away from the faintest radio contours which overlaps soft X-ray emission detected by ROSAT. Radio observations would be required to establish the nature of this structure.

Acknowledgements. It is a pleasure to thank R. G. Strom for his comments and for providing the $49 \mathrm{~cm}$ radio data. We are further indebted to the referee whose pertinent remarks helped clarify, and enhance the scope of this paper. Skinakas Observatory is a collaborative project of the University of Crete, the Foundation for Research and Technology-Hellas and the Max-Planck-Institut für Extraterrestrische Physik. This work has been supported by a P.EN.E.D. program of the General Secretariat of Research and Technology of Greece. J.V. acknowledges support through an Alexander von Humboldt Fellowship. This research has made use of data obtained through the High Energy Astrophysics Science Archive Research Center Online Service, provided by the NASA/Goddard Space Flight Center. 


\section{References}

Angerhofer, P. E., Strom, R. G., Velusamy, T., \& Kundu, M. R. 1981, A\&A, 94, 313

van den Bergh, S. 1980, PASP, 92, 768

Blair, W. P., Kirshner, R. P., Fesen, R. A., \& Gull, T. R. 1984, ApJ, 282, 161

Blair, W. P., Fesen, R. A., \& Becker, R. H. 1988, AJ, 96, 1011

Cioffi, D. F., McKee, C. F., \& Bertschinger, E. 1988, ApJ, 334, 252

Clifton, T. R., Backer, D. C., Foster, R. S., et al. 1987, IAU Circ., No. 4422

Cox, D. P., \& Raymond, J. C. 1985, ApJ, 298, 651

Downes, D. 1970, Ph.D. Thesis, Harvard University

Fesen, R. A., Shull, J. M., \& Saken, J. M. 1988, Nature, 334, 229

Halm, I. 1996, MPE report 263, ed. H. U. Zimmerman, J. Trümper, \& H. Yorke

Hartigan, P., Raymond, J., \& Hartmann, L. 1987, ApJ, 316, 323

Hester, J. J. 1987, ApJ, 314, 187

Hester, J. J., \& Kulkarni, S. R. 1989, ApJ, 340, 362

Kaler, J. B. 1976, ApJS, 31, 517

Koo, B. C., Yun, M. S., Ho, P. T., \& Lee, Y. 1993, ApJ, 417, 196
Koo, B. C., Reach, W. T., Heiles, C., Fesen, R. A., \& Shull, M. 1990, ApJ, 364, 178

Lynds, B. T. 1965, ApJS, 12, 163

Maciejewski, W., \& Cox, D. P. 1999, ApJ, 511, 972

Mantovani, F., Reich, W., Salter, C. J., \& Tomasi, P. 1985, A\&A, 145, 50

Mavromatakis, et al. 2000, under preparation

Raymond, J. C., Hester, J. J., Cox, D., et al. 1988, ApJ, 324, 869

Raymond, J. C. 1979, ApJS, 39, 1

Ryter, C., Cesarsky, C. J., \& Audouze, J. 1975, ApJ, 198, 103

Safi-Harb, S., Ögelman, H., \& Finley, J. 1995, ApJ, 439, 722

Strom, R. G. 1987, ApJ, 310, L103

Strom, R. G., \& Stappers, B. W. 2000, Pulsar Astronomy - 2000 and Beyond, ed. M. Kramer, N. Wex, \& R. Wielebinski

Wang, Z. R., \& Seward, F. D. 1984, ApJ, 285, 607

Wheelock, S. L., Gautier, T. N., Chillemi, J., et al. 1994, IRAS sky survey atlas, Explanatory supplement

Whitehead, M. J., Meaburn, J., \& Clayton, C. A. 1989, MNRAS, 237, 1109

Whitford, A. 1958, AJ, 63, 201

Velusamy, T., \& Kundu, M. R. 1974, A\&A, 32, 375 